\title{
Efecto del nivel de suplementación con concentrado sobre el consumo voluntario y comportamiento ingestivo en vacas lecheras a pastoreo primaveral ${ }^{\#}$
}

\author{
Effect of the level of concentrate supplementation on the voluntary \\ intake and feeding behaviour of dairy cows on spring grazing \\ C Riquelme, RG Pulido* \\ Instituto de Ciencia Animal, Facultad de Ciencias Veterinarias, Universidad Austral de Chile, Valdivia, Chile.
}

\begin{abstract}
SUMMARY
The purpose of this study was to evaluate the effect of different levels of concentrate supplementation on grazing behaviour of dairy cows fed spring pasture. The study was conducted at "Vista Alegre" Experimental Farm, from September to November 2005. Twenty eight spring calving Friesian cows were assigned to four experimental treatments: SP, grazing alone; PSC3, pasture plus $3 \mathrm{~kg} / \mathrm{d}$ of concentrate; PSC6, pasture plus $6 \mathrm{~kg} / \mathrm{d}$ and PSC9, pasture plus $9 \mathrm{~kg} / \mathrm{d}$ of concentrate. Dry matter intake was estimated using chromic oxide capsules of automatic liberation as an indigestible marker. Individual measurement of feeding behaviour was carried out in two opportunities, recording the grazing, ruminating, laying, standing, walking and milking activities every ten minutes during 24 hours. The cows were supplemented twice a day and were managed under a strip grazing system on pasture consisting mainly of perennial ryegrass. The pasture intake decreased $(\mathrm{P}<0.001)$ in all treatments with concentrate supplementation $(17.6 ; 15.2 ; 11.4$ and $7.7 \mathrm{~kg}$ dry matter/day, for PS, PSC3, PSC6 and PSC9, respectively); meanwhile total dry matter intake was decreased by supplementation only in treatment PSC9 $(\mathrm{P}<0,05)$. The substitution rate was $0.92 ; 1.17$ and $1.25 \mathrm{~kg}$ dry matter of pasture/kg dry matter of concentrate for PSC3, PSC6 and PSC9 treatments, respectively. Total grazing time decreased (447, 414, 413 and $381 \mathrm{~min} /$ day, for PS, PSC3, PSC6, and PSC9, respectively) and total ruminating time increased (463, 473, 495 and $501 \mathrm{~min} /$ day, for PS, PSC3, PSC6, and PSC9, respectively), as a result of supplementation, but it was significant only between PS and PSC9. Unsupplemented cows and cows receiving $3 \mathrm{~kg} / \mathrm{day}$ had a higher bite rate $(\mathrm{P}<0.001)$ than supplemented cows receiving 6 and $9 \mathrm{~kg} / \mathrm{day}$. The results suggest that high levels of supplementation $(9 \mathrm{~kg} / \mathrm{day})$ decrease pasture dry matter intake and grazing time, while increasing ruminating time, but have no effect on total dry matter intake.
\end{abstract}

Palabras clave: vaca lechera, comportamiento ingestivo, concentrado, pastoreo.

Key words: dairy, ingestive behaviour, concentrate, pasture.

\section{INTRODUCCION}

La principal fuente de alimentación de vacas destinadas a producción láctea, en el sur de Chile, es la utilización directa de praderas permanentes (Balocchi y col 2002); sin embargo, el bajo consumo de materia seca y energía metabolizable y la falta de sincronía en el rumen entre la energía y la proteína cruda aportada por el forraje, son los principales factores que limitan la producción de leche en pastoreo (Stockdale 2000). La investigación ha mostrado que praderas de alta calidad en primavera y bien manejadas son capaces de sustentar producciones de leche hasta de 30 L por día en vacas sin suplementación (Bargo y col 2003). Por lo tanto, la inclusión en la dieta de alimentos suplementarios, como concentrado, son requeridos para incrementar el consumo de energía y de materia seca total (McGilloway y Mayne 1996).

\footnotetext{
Aceptado: 08.03.2008.

\# Financiado por Proyecto FONDECYT 1030331

* Casilla 567, Valdivia, Chile; rpulido@uach.cl
}

Es aceptado que factores del animal, de la pradera y del alimento suplementario afectan la tasa de sustitución de pradera por concentrado, donde se pueden incluir la oferta de pradera por vaca por día, la cantidad de concentrado, las características nutritivas de la pradera y del concentrado y el estado de lactancia (Kellaway y Porta 1993). Entre los factores nombrados, la cantidad de pradera por vaca por día y el nivel de concentrado tienen un gran efecto sobre la tasa de sustitución (Peyraud y Delaby 2001). Varios estudios han informado que la tasa de la oferta de pradera por vaca por día y el nivel de concentrado afectan el consumo de pradera y el comportamiento en pastoreo (Arriaga-Jordan y Holmes 1986, Pulido y Leaver 2001, Sairanen y col 2006). Si bien la literatura es abundante en relación a la suplementación de vacas a pastoreo (Bargo y col 2003), es necesario recabar más información respecto del efecto de una alta oferta de forraje y su relación con distintos niveles de concentrado, sobre el consumo de alimento y comportamiento alimenticio en pastoreo de vacas de alta producción a comienzo de la lactancia (Sayers y col 2003).

El objetivo de este trabajo fue evaluar el efecto de distintos niveles de suplementación con concentrado 
sobre el consumo voluntario y comportamiento ingestivo de vacas lecheras a pastoreo primaveral con oferta $a d$ libitum de pradera.

\section{MATERIAL Y METODOS}

El ensayo se efectuó en el predio experimental "Vista Alegre", de propiedad de la Universidad Austral de Chile, ubicado $6 \mathrm{~km}$ al norte de la ciudad de Valdivia $\left(39^{\circ} 47^{\prime}\right.$ 46 " y $39^{\circ} 48^{\prime} 54^{\prime \prime}$ de Latitud Sur y $73^{\circ} 12^{\prime} 24^{\prime \prime}$ y $73^{\circ}$ 13' 13" de Longitud Oeste), desde septiembre hasta noviembre de 2005. El suelo corresponde a la serie Valdivia (Typic Hapludand), de topografía ligeramente ondulada y sin problemas de drenaje. Se utilizaron 28 vacas Frisón Negro, que al inicio del estudio promediaban 56,4 $\pm 4,7$ días de lactancia, 4,1 $\pm 0,1$ partos, una producción láctea de 30,7 \pm 0,7 litros, una condición corporal de 2,3 $\pm 0,1$ y un peso vivo de $538 \pm 13$ kilos (promedio $\pm \mathrm{DE}$ ). Las vacas fueron bloqueadas en 4 grupos de 7 vacas cada uno, de acuerdo a la producción de leche, días en leche y peso vivo. Los grupos se asignaron al azar a 4 tratamientos dietarios: SP, sólo pastoreo; PSC3, pastoreo más suplementación con $3 \mathrm{~kg} /$ día de concentrado; PSC6, pastoreo más suplementación con $6 \mathrm{~kg} /$ día de concentrado y PSC9, pastoreo más suplementación con $9 \mathrm{~kg}$ /día de concentrado, en un diseño continuo.

La pradera correspondió a una del tipo permanente mejorada, constituida principalmente por ballica perenne (Lolium perenne), con uniformidad en edad y manejo, ubicada entre 300 y 500 metros de la sala de ordeña. Se utilizó un concentrado peletizado especialmente formulado para el ensayo, compuesto por un $94 \%$ de cebada, $4 \%$ de afrecho de raps y un $2 \%$ de melaza, siendo ofrecido en parcialidades iguales en cada ordeña según tratamiento. Sales minerales se ofrecieron junto al concentrado y también a libre disposición en saleros en el camino a la sala de ordeña. El agua fue ofrecida a libre disponibilidad, tanto en bebederos móviles en los potreros como en bebederos que se encuentran en la sala de ordeña. La ordeña se realizó 2 veces al día, empezando a las 6:30 y 17:00 horas.

Las vacas se manejaron bajo un sistema de pastoreo rotativo, en donde cada tratamiento pastoreó en forma independiente su propia superficie de pastoreo y teniendo una rotación de potrero 2 veces al día, inmediatamente postordeña. A cada vaca se le otorgó una disponibilidad de pradera de, al menos, 36 kg/día de materia seca (MS). Se calculó superficie de pastoreo para cada tratamiento por el método de medición de la altura de la pradera prepastoreo, a través de una regresión lineal entre la altura de la pradera, obtenida cada día y para cada potrero, y la disponibilidad de la MS, ecuación previamente calculada y actualizada a lo largo del estudio, de acuerdo a lo señalado por Hodgson (1990). El mismo método se empleó para el postpastoreo, cuantificando así el residuo dejado por cada tratamiento.
El consumo voluntario se estimó a partir del consumo de pradera y del concentrado que cada vaca tenía. El consumo de concentrado se calculó midiendo la diferencia entre lo ofrecido y lo rechazado por los animales. El consumo de pradera se estimó a través del método de rendimiento fecal y digestibilidad de la dieta, descrito por Le Du y Penning (1982). A cada vaca se le suministró, vía oral, una cápsula de óxido de cromo como marcador indigestible de liberación automática (Captec®, NZ), liberando 1,7 g/día de éste. Transcurrida la primera semana, en la que ocurrió la estabilización de la excreción del marcador a través de las heces, se recolectaron muestras de fecas durante 2 períodos de 1 semana de duración, 2 veces al día, previo a cada ordeña, para así poder realizar la determinación de la concentración de óxido de cromo.

El comportamiento ingestivo se realizó en 2 oportunidades por cuatro observadores entrenados, observándose la actividad de cada uno de los animales cada 10 minutos, durante $24 \mathrm{~h}$. Las vacas fueron identificadas individualmente con un número visible pintado en cada flanco. Las actividades registradas fueron: comiendo, rumiando echada, rumiando parada, echada, parada, caminando y bebiendo. La tasa de bocados se estimó registrando la cantidad de mordiscos dados por cada vaca en 60 segundos, lo que se realizó 5 veces para cada animal, distribuidas en los ciclos de pastoreo. Si entre cada bocado transcurrían más de 10 segundos la medición se anulaba y se reiniciaba todo el proceso.

\section{ANALISIS DE MUESTRAS}

Quincenalmente se tomó una muestra compuesta de las praderas pastoreadas por cada tratamiento y del concentrado utilizado, las que fueron analizadas en el Laboratorio de Nutrición Animal de la Universidad Austral de Chile. El contenido de MS se determinó mediante horno de ventilación a $60{ }^{\circ} \mathrm{C}$ por 48 horas, o estufa a $105^{\circ} \mathrm{C}$ por 12 horas (Bateman 1970); las cenizas totales (CT) mediante calcinación en mufla a $550-600{ }^{\circ} \mathrm{C}$ por 5 horas (Bateman 1970); la energía metabolizable (EM) mediante regresión a partir del valor "D” (Garrido y Mann 1981); el extracto etéreo (EE) mediante análisis proximal (Bateman 1970); la fibra detergente neutra (FDN) por digestión con detergente neutro (Van Soest y col 1991); la fibra detergente ácida (FDA) a partir de digestión con detergente ácido (AOAC 1996); y la proteína bruta (PC) mediante microkjeldhal (Bateman 1970). Las muestras de pradera se tomaron desde los $7 \mathrm{~cm}$ sobre el suelo, utilizando una tijera, para simular lo consumido por las vacas. La digestibilidad de los alimentos fue calculada a partir del valor "D", mediante el método de digestibilidad in vitro con licor ruminal de Tilley y Terry (1963) modificado por Goering y Van Soest (1972). La cuantificación de la concentración de óxido de cromo $\left(\mathrm{Cr}_{2} \mathrm{O}_{3}\right)$ en las heces se realizó mediante el método descrito por Bateman (1970). 


\section{ANALISIS ESTADISTICO}

Los datos fueron sometidos a un análisis de varianza aleatorio continuo mediante la utilización de medidas repetidas, con un nivel de significancia del $5 \%$, empleando el programa MINITAB $囚$ Release $14.20 .{ }^{1}$ La comparación de las diferencias entre tratamientos se realizó mediante la prueba de Tukey. El modelo estadístico fue $y_{i j k l}=\mu+T_{\mathrm{i}}+$ $C_{\mathrm{ij}}+P_{\mathrm{k}}+T P_{\mathrm{ik}}+e_{j k l .}$, donde: $y_{i j k l}=$ variables dependientes; $\mu$ = intercepto general; $T_{\mathrm{i}}=$ el efecto fijo del tratamiento; $C_{\mathrm{ij}}=$ el efecto fijo de la vaca; $P_{\mathrm{k}}=$ el efecto fijo del período kth de observación; $T P_{\mathrm{ik}}=$ el efecto fijo de la intersección entre el tratamiento ith y el período kth de observación; $e_{j k l}=$ error residual.

\section{RESULTADOS}

\section{CONDICIONES AMBIENTALES}

La temperatura media en los meses de septiembre, octubre y noviembre fue de $9,7,11,3$ y $13,8^{\circ} \mathrm{C}$, respectivamente, comportándose de manera similar a los valores promedio de los últimos 40 años. Las precipitaciones registradas durante septiembre fueron $148,6 \mathrm{~mm}$, en octubre $61,7 \mathrm{~mm}$ y en noviembre $277,5 \mathrm{~mm}$, lo que comparado a los valores promedio indican un déficit del $21,4 \%$ y $58,8 \%$ durante septiembre y octubre, y un superávit de 165,6\% en noviembre. La primera observación del comportamiento ingestivo se realizó del 9 al 10 de octubre, no registrándose precipitaciones, y con una temperatura media de $12^{\circ} \mathrm{C}$. La segunda medición fue del 19 al 20 de noviembre, cuantificándose $4,8 \mathrm{~mm}$ caídos y $13,5^{\circ} \mathrm{C}$ de temperatura media.

\section{CARACTERISTICAS DE LOS ALIMENTOS Y}

MANEJO DE LA PRADERA

El cuadro 1 muestra la composición química promedio de muestras quincenales de los alimentos utilizados en el estudio, observándose en el concentrado un alto nivel de digestibilidad y alta concentración de energía metabolizable. En la pradera destaca su alta concentración energética $(2,87$ $\mathrm{Mcal} / \mathrm{kg}$ ) y su concentración promedio de FDN (51\%). En el cuadro 2 se aprecia que la presión de pastoreo de $36,7 \mathrm{~kg}$ MS/vaca/día estuvo sobre el mínimo establecido para este ensayo y que la eficiencia de utilización de la pradera fue similar para los tratamientos suplementados.

\section{CONSUMO DE ALIMENTOS Y}

\section{COMPORTAMIENTO EN PASTOREO}

En el cuadro 3 se muestra la disminución significativa en el consumo de pradera a medida que se aumenta el nivel de suplementación. El consumo total de MS sólo disminuyó significativamente $(\mathrm{P}<0,01)$ entre PSC3 y PSC9. La tasa de sustitución fue de 0,92, 1,17 y 1,25 kg MS de pradera por kg MS de concentrado para los tratamientos PSC3, PSC6 y PSC9, respectivamente. En el tiempo de pastoreo y rumia se observa una disminución significativa entre el tratamiento SP y PSC9. No se observaron diferencias significativas $(\mathrm{P}>0,05)$ en la tasa de consumo.

\section{DISCUSION}

Las praderas permanentes fertilizadas de la zona sur de Chile, durante primavera, poseen un $15,2 \%$ de MS; $11,5 \%$

Cuadro 1. Composición de los alimentos utilizados en el experimento.

Composition of experimental feeds.

\begin{tabular}{lcccc}
\hline & \multicolumn{2}{c}{ Concentrado* } & \multicolumn{2}{c}{ Pradera } \\
\cline { 2 - 5 } & \multicolumn{2}{c}{$\mathrm{X} \pm \mathrm{DE}$} & & $\mathrm{X} \pm \mathrm{DE}$ \\
\hline Materia seca (MS)\% & 87,3 & 0,96 & 15,9 & 3,02 \\
Cenizas totales (CT)\% & 3 & 0,55 & 7 & 2,46 \\
Proteína bruta (PC)\% & 13 & 0,98 & 22,4 & 4,67 \\
Extracto etéreo (EE)\% & 2,6 & 0,58 & 3,1 & 0,92 \\
Energía metabolizable (EM) Mcal/kg & 3,13 & 0,10 & 51 & 0,15 \\
Fibra detergente neutra (FDN)\% & 22,3 & 2,83 & 25,3 & 8,36 \\
Fibra detergente ácida (FDA)\% & 6,5 & 1,54 & 78 & 4,16 \\
IVDMD $\%$ & 87,6 & 0,74 & 1,65 & \\
\hline
\end{tabular}

1 IVDMD = Digestibilidad de la materia seca in vitro.

* = Concentrado formulado especialmente para este experimento por IANSAGRO S.A.

\footnotetext{
1 MINITAB Release 12 Statistical Software (1998).
} 
Cuadro 2. Manejo del pastoreo.

Pasture management.

\begin{tabular}{|c|c|c|c|c|c|c|}
\hline & \multicolumn{6}{|c|}{ Tratamientos } \\
\hline & SP & PSC 3 & PSC 6 & PSC 9 & $\mathrm{X}$ & $\mathrm{DE}$ \\
\hline Carga animal (vacas/ha) & - & - & - & - & 3,08 & - \\
\hline Presión de pastoreo (kg MS/vaca/día) & 36,8 & 36,5 & 36,9 & 36,7 & 36,7 & 6,3 \\
\hline Área $\left(\mathrm{m}^{2} / \mathrm{vaca} /\right.$ día $)$ & 120,7 & 119,8 & 120,8 & 120,4 & 120,4 & 15,6 \\
\hline Disponibilidad (kg MS/ha)* & 2.893 & 2.893 & 2.893 & 2.893 & 2.893 & 417 \\
\hline Residuo (kg MS/ha)* & 1.566 & 1.757 & 1.819 & 1763 & 1.726 & 239 \\
\hline Eficiencia utilización (\%) & 45,9 & 39,3 & 37,1 & 39,0 & 40,3 & 3,8 \\
\hline Altura promedio prepastoreo (plato) ${ }^{1}$ & - & - & - & - & 24,5 & 4,7 \\
\hline Altura promedio postpastoreo (plato) ${ }^{1}$ & - & - & - & - & 17,1 & 2,1 \\
\hline
\end{tabular}

${ }^{1}$ Altura comprimida con plato medidor.

$*$ = Ecuación de disponibilidad de pradera; $\mathrm{y}=737,5+88,1 \mathrm{x}\left(\mathrm{r}^{2}=0,85\right) ; \mathrm{y}=$ Fitomasa disponible, $\mathrm{x}=$ altura comprimida del plato.

Cuadro 3. Consumo de alimento y comportamiento en pastoreo de vacas lecheras sin suplementación y suplementadas con distintos niveles de concentrado.

Food intake and grazing behavior of dairy cows that were unsupplemented and supplemented with diferents levels of concentrate.

\begin{tabular}{lccccc}
\hline & \multicolumn{5}{c}{ Tratamientos } \\
\cline { 2 - 6 } & SP & PSC 3 & PSC 6 & PSC 9 & P \\
\hline Consumo de pradera (kg MS/vaca/día) & $17,6^{\mathrm{a}}$ & $15,2^{\mathrm{b}}$ & $11,4^{\mathrm{c}}$ & $7,7^{\mathrm{d}}$ & 0,001 \\
Consumo total MS (kg MS/vaca/día) & $17,6^{\mathrm{ab}}$ & $17,8^{\mathrm{a}}$ & $16,6^{\mathrm{ab}}$ & $15,6^{\mathrm{b}}$ & 0,03 \\
Tasa de sustitución1 & - & 0,92 & 1,17 & 1,25 & - \\
Consumo de FDN (kg MS/vaca/día) & $9^{\mathrm{a}}$ & $8,4^{\mathrm{ab}}$ & $7,1^{\mathrm{c}}$ & $5,8^{\mathrm{d}}$ & 0,001 \\
& & & & & \\
Actividades & & & & & \\
Pastoreo (min/día) & $447,1^{\mathrm{a}}$ & $413,6^{\mathrm{ab}}$ & $412,9^{\mathrm{ab}}$ & $380,7^{\mathrm{b}}$ & 0,001 \\
Rumia (min/día) & $462,9^{\mathrm{a}}$ & $472,9^{\mathrm{ab}}$ & $495^{\mathrm{ab}}$ & $501,4^{\mathrm{b}}$ & 0,013 \\
Otras actividades total (min/d) & $530^{\mathrm{a}}$ & $553,6^{\mathrm{a}}$ & $532,1^{\mathrm{a}}$ & $552,1^{\mathrm{a}}$ & 0,28 \\
Tasa de consumo (g MS/min) & $39,4^{\mathrm{a}}$ & $36,9^{\mathrm{a}}$ & $27,6^{\mathrm{a}}$ & $20,4^{\mathrm{a}}$ & 0,237 \\
Tasa de bocado & $58^{\mathrm{a}}$ & $55^{\mathrm{a}}$ & $52^{\mathrm{b}}$ & $51^{\mathrm{b}}$ & 0,001 \\
Bocados diarios $^{2}$ & $25.932^{\mathrm{a}}$ & $22.748^{\mathrm{a}}$ & $21.471^{\mathrm{a}}$ & $19.416^{\mathrm{b}}$ & 0,004 \\
\hline
\end{tabular}

${ }^{1} \mathrm{~kg}$ MS pradera/kg MS concentrado.

${ }^{2}$ bocados/min.

de CT; $17,8 \%$ de PC; un 3,2\% de EE; 27,9\% de FDA y 2,55 $\mathrm{Mcal} / \mathrm{kg}$ (FIA-UACH 1995). Si se comparan los valores referenciales recién mencionados con los del cuadro 1 , se tienen valores similares para la MS $(15,2$ vs. 15,9$)$, EE $(3,2$ vs. 3,1$)$ y FDA ( 27,9 vs. 25,3$)$. Valores disímiles son encontrados para las CT (11,5 vs. 7$)$, PC (17,8 vs. 22,4$)$ y EM (2,55 vs. 2,87). Entonces, la pradera presentó un alto valor nutritivo durante el estudio, especialmente para los factores más limitantes como son la EM y la PC. La FDN para una pradera de buena calidad, según Kolver y Müller (1998), es aquella que fluctúa entre un 40 y 50\%, siendo en el caso de este ensayo de un 51\%, reflejando concordancia con lo reportado anteriormente y con ensayos anteriores de Pulido y col (2007), realizados en similares condiciones.

En el cuadro 2 se observa que la carga animal instantánea fue de 3,08 , valor usualmente encontrado en este tipo de ensayos (Pulido y col 2006, 2007), debido al uso de un elevado número de vacas en una superficie relativamente pequeña de hectáreas, a causa de una alta disponibilidad de forraje primaveral. La presión de pastoreo y el área por vaca fue similar entre tratamientos $(\mathrm{P}>0,05)$. La 
disponibilidad y el residuo observados son similares a lo descrito por Pulido y col (2006) en años anteriores en el mismo predio.

La eficiencia de utilización de la pradera fue baja e inferior al $40 \%$ en todos los tratamientos, salvo en el caso de SP, en que superó ese porcentaje. McGilloway y Mayne (1996) señalan que estos valores bajos son totalmente esperables para este tipo de ensayos, donde la oferta de pradera tiene como objetivo no limitar el consumo voluntario de la vaca, lo cual se logra sólo en cortos períodos durante la primavera, cuando la oferta y la calidad de la pradera son muy buenas.

Es aceptado que diversos factores de la pradera afectan el consumo de MS (Rook 2000), incluyendo la cantidad de pradera previa al pastoreo (kg MS/ha) y la disponibilidad de pradera (cantidad de pastura ofrecida por vaca; $\mathrm{kg} \mathrm{MS/vaca/día).} \mathrm{En} \mathrm{este} \mathrm{ensayo} \mathrm{la} \mathrm{alta} \mathrm{oferta} \mathrm{de} \mathrm{pradera}$ en promedio de $36 \mathrm{~kg} \mathrm{MS/vaca/día} \mathrm{y} \mathrm{alta} \mathrm{calidad} \mathrm{de} \mathrm{ésta}$ permitió que el consumo de pradera en las vacas sin suplementación alcanzara a los 17,6 kg de materia seca, correspondiendo a 3,3\% del peso vivo, valor que está de acuerdo a experimentos con condiciones de pradera y animales similares a éstos (Bargo y col 2003).

El principal objetivo de la suplementación de vacas en pastoreo es aumentar el consumo total de materia seca y el consumo de energía, sobre aquel que se logra con dietas sólo en base a pradera (Stockdale 2000, Peyraud y Delaby 2001). Sin embargo, cuando las vacas en pastoreo reciben suplementos, el consumo de MS de pradera generalmente disminuye, lo cual es conocido como tasa de sustitución (Kellaway y Porta 1993). En este ensayo, la suplementación disminuyó significativamente el consumo de forraje en los tratamientos suplementados (cuadro 3), confirmando las conclusiones obtenidas por diferentes autores (Jones-Endsley y col 1997, Reis y Combs 2000, Balocchi y col 2002). La tasa de sustitución (TS) fue de $0,92,1,17$ y $1,25 \mathrm{~kg}$ MS pradera/kg MS de concentrado para los tratamientos PSC3, PSC6 y PSC9, respectivamente, manifestando el impacto que tuvo la suplementación en la disminución del consumo de pradera $(\mathrm{P}<0,05)$, alcanzando a 2,4, 6,2 y 9,9 kg/MS/día para PSC3, PSC6 y PSC9 versus $\mathrm{SP}$, respectivamente. La magnitud de la disminución en el consumo de pradera explica el hecho de que el consumo total de MS fuera similar entre tratamientos, a excepción del mayor consumo logrado por PSC 3 comparado con PSC9. Bajo las condiciones de este ensayo (alta oferta de pradera de buena calidad y superficie de pastoreo similar para los tratamientos), la suplementación a altos niveles de concentrado de vacas en pastoreo primaveral afecta negativamente el consumo de pradera e impide aumentar el consumo total de materia seca de éstas.

El tiempo de pastoreo diario del tratamiento SP es menor si se compara a su símil en trabajos realizados por Bargo y col (2002) y por Pulido y col (2006). Peyraud y col (1996) señalaron que los bajos tiempos de pastoreo registrados en vacas manejadas en pastoreo en franjas, reflejarían la habilidad de los animales de anticipar el cambio a una nueva franja, por lo que el tiempo total de pastoreo disminuiría dado que las vacas preferirían esperar la nueva franja aportada después de cada ordeña. Es aceptado que el efecto de los suplementos en el consumo de materia seca del forraje es mediado a través de modificaciones en el comportamiento alimenticio en pastoreo y especialmente a través de la tasa de consumo y en el tiempo de pastoreo (Rook 2000, Pulido y Leaver 2001). El tiempo de pastoreo disminuyó por efecto de la suplementación en promedio en 9,6 minutos por $\mathrm{kg}$ de concentrado aportado, tiempo mayor al encontrado por Balocchi y col (2006), pero consistente con las reducciones significativas señaladas por Arriaga- Jordan y Holmes (1986) y por Rook y col (1994), y con las reducciones numéricas presentadas por Gibb y col (2002).

El tiempo de rumia aumentó significativamente en 4,3 minutos por kg de concentrado, entre los tratamientos SP y PSC9, pero no hubo efecto cuando se suplementó con niveles bajos o moderado de concentrado. Concordando con nuestros resultados, Balocchi y col (2002) y Sayers y col (2003) señalan que no existen diferencias en el tiempo total de rumia en vacas lecheras recibiendo hasta $6 \mathrm{~kg}$ de concentrado al día. Sin embargo, Bargo y col (2002) y Sayers y col (2003) reportan una disminución en el tiempo de rumia por efecto de una alta suplementación con concentrado, nivel de concentrado similar al nivel máximo de este ensayo sería resultante de una reducción del pH ruminal. Si bien en este ensayo también se reportó una disminución en el pH ruminal (Gebauer y col 2006), éste no habría producido un efecto negativo en el tiempo destinado a la rumia, ya que las vacas del tratamiento PSC9 tuvieron un menor tiempo de pastoreo $(\mathrm{P}>0,05)$, $\mathrm{y}$ un significativo menor deseo de pastorear medido como tasa de bocados $(\mathrm{P}<0,001)$ o número de bocados diarios $(\mathrm{P}<0,004)$, quedando más tiempo para rumiar lo consumido. La tasa de bocados y el número de bocados diarios disminuyó por efecto de la suplementación, pero sólo significativamente entre PS y el tratamiento PSC9. Lo anterior da cuenta del efecto de que elevados niveles de suplementación pueden reflejar diferencias entre tratamientos en la motivación animal por pastorear, deprimiendo los parámetros anteriormente señalados.

No hubo diferencias significativas $(P>0,05)$ en el tiempo total destinado a otras actividades, siendo los valores obtenidos superiores a los de la literatura consultada (Leiva 2006, Pulido y col 2006), lo que se explicaría por el bajo tiempo de pastoreo y rumia al comparar con otros trabajos similares.

Con la información recabada durante el experimento se puede concluir que distintos niveles de suplementación con concentrado, utilizando praderas de elevada calidad nutritiva y con gran disponibilidad de forraje, disminuyen 
el consumo de materia seca de la pradera, sin incrementar el consumo total de materia seca. La suplementación con $9 \mathrm{~kg}$ de concentrado disminuye el tiempo de pastoreo y aumenta el tiempo de rumia.

\section{RESUMEN}

Se realizó un experimento de pastoreo con el objetivo de evaluar el efecto de distintos niveles de suplementación con concentrado, sobre el comportamiento ingestivo de vacas lecheras a pastoreo primaveral con oferta abundante de pradera. El estudio se efectuó en la Estación Experimental "Vista Alegre", desde el 19 de septiembre hasta el 20 de noviembre del 2005. Se usaron 28 vacas Frisón Negro, estableciéndose 4 tratamientos: SP, sólo pastoreo; PSC3, pastoreo más $3 \mathrm{~kg}$ de concentrado/día; PSC6, pastoreo más $6 \mathrm{~kg}$ de concentrado/ día y PSC9 pastoreo más $9 \mathrm{~kg}$ de concentrado/día. El consumo de materia seca se estimó usando cápsulas de óxido de cromo como marcador indigestible. El comportamiento ingestivo se observó en 2 oportunidades, registrando en las vacas individualmente las actividades; tiempo de pastoreo, tiempo de rumia, tiempo paradas o echadas y la tasa de bocados. El consumo de pradera disminuyó $(\mathrm{P}<0,001)$ en todos los tratamientos $(17,6 ; 15,2 ; 11,4$ y $7,7 \mathrm{~kg}$ MS/día, para PS, PSC3, PSC6 y PSC9, respectivamente), mientras que el consumo total de materia seca no se modificó por efecto de la suplementación. La tasa de sustitución fue 0,$92 ; 1,17$ y $1,25 \mathrm{~kg}$ MS pradera/kg MS concentrado, para PSC3, PSC6 y PSC9, respectivamente. El tiempo de pastoreo disminuyó $(\mathrm{P}<0,001)$ y el tiempo de rumia aumentó, pero sólo entre PS y PSC9. La tasa de bocados fue similar para PS y PSC3, pero mayor $(\mathrm{P}<0,001)$ que en los tratamientos PSC6 y PSC9. Bajo las condiciones de este ensayo, se concluye que el consumo de materia seca de pradera disminuye al aumentar el nivel de suplementación, sin modificar el consumo total de materia seca. Por su parte, altos niveles de suplementación con concentrado ( $9 \mathrm{~kg} /$ día) disminuyen el tiempo de pastoreo, la tasa de bocados y aumentan el tiempo de rumia, reflejando los efectos tratamientos en motivación animal por pastorear.

\section{REFERENCIAS}

AOAC, Association of official analytical chemists. 1996. Official methods of analysis. $16^{\text {th }}$ ed. AOAC, Arlington, VA, USA.

Arriaga-Jordan CM, W Holmes. 1986. The effect of cereal concentrate supplementation on the digestibility of herbage based diets for lactating dairy cows. J Agric Sc 106, 581-592.

Balocchi O, R Pulido, J Fernández. 2002. Comportamiento de vacas lecheras en pastoreo con y sin suplementación de concentrado. Agri Téc 62, 87-98.

Bargo F, LD Muller, JE Delahoy, TW Cassidy. 2002. Milk response to concentrate supplementation of high producing dairy cows grazing at two pasture allowances. J Dairy Sci 85, 1777-1792.

Bargo F, LD Muller, ES Kolver, JE Delahoy. 2003. Invited review: Production and digestion of supplemented dairy cows on pasture. J Dairy Sci 86, 1-42.

Bateman J. 1970. Nutrición animal. Manual de métodos analíticos. Centro Regional de Ayuda Técnica. México, Pp 468.

FIA-UACH. 1995. Composición de Alimentos para el Ganado en la Zona Sur. Universidad Austral de Chile. Fundación Fondo de Investigación Agropecuaria (eds), Ministerio de Agricultura, Editorial Universitaria, Valdivia, Chile.

Garrido O, E Mann. 1981. Composición química, digestibilidad y valor energético de una pradera permanente de pastoreo a través del año. Memoria de título, Facultad de Ciencias Agrarias, Universidad Austral de Chile, Valdivia, Chile.

Gebauer I, M Leiva, F Wittwer, R Pulido. 2006. Nivel de suplementación con concentrado en vacas lecheras a pastoreo primaveral.
II Evaluación del pH y nitrógeno amoniacal en líquido ruminal. Resúmenes del XX Congreso Panamericano de Ciencias Veterinarias, Santiago, Chile.

Gibb MJ, CA Huckle, R Nuthall. 2002. Effects of level of concentrate supplementation on grazing behaviour and performance by lactating dairy cows grazing continuously stocked grass swards. Anim Sci 74, 319-335.

Goering H, P Van Soest. 1972. Análisis de fibra de forraje. Universidad Agraria La Molina, Lima, Perú.

Hodgson J. 1990. Grazing Management. Science into Practice. Longman Scientific and Technical. Essex, England.

Jones-Endsley JM, MJ Cecava, TR Johnson. 1997. Effects of dietary supplementation on nutrient digestion and the milk yield of intensively grazed lactating dairy cows. J Dairy Sci 80, 3283-3292.

Kellaway R, S Porta. 1993. Feeding concentrates: Supplements for dairy cows. Dairy Research and Development Corporation, Glen Iris, Victoria, Australia.

Kolver ES, LD Muller. 1998. Performance and nutrient intake of high producing Holstein cows consuming pasture or a total mixed ration. J Dairy Sci 81, 1403-1411.

Le Du YLP, PD Penning. 1982. Animal based techniques for estimating herbage intake. In: Leaver JD (ed). Herbage intake handbook. The British Grassland Society, UK, Pp 77-93.

Leiva M. 2006. Evaluación del aumento de la frecuencia de suplementación con concentrado en vacas lecheras en pastoreo primaveral sobre el consumo voluntario y comportamiento alimenticio. Memoria de título, Escuela de Medicina Veterinaria, Universidad Austral de Chile, Valdivia, Chile.

McGilloway DA, CS Mayne. 1996. The importance of grass availability for the high genetic merit dairy cows. In: Garnsworthy PC, Wiseman J, Haresign W (eds). Recent Advances in Animal Nutrition. Nottingham University Press, Nottingham, UK, Pp 135-169.

Peyraud JL, EA Comeron, M Wade, G Lemaire. 1996. The effect of daily herbage allowance, herbage mass and animal factors upon herbage intake by grazing dairy cows. Animal Zootech 45, 201-217.

Peyraud JL, L Delaby. 2001. Ideal concentrate feeds for grazing dairy cows responses to supplementation in interaction with grazing management and grass quality. In: Garnsworthy PC, Wiseman J (eds). Recent Advances in Animal Nutrition. Notthingham University Press, Notthingham, UK.

Pulido RG, JD Leaver. 2001. Quantifying the influence of sward height, concentrate level, and initial milk yield on the milk production and grazing behaviour of continuously stocked dairy cows. Grass Forr Sci 56, 57-67.

Pulido RG, E Felmer, A Hinostroza. 2006. Efecto del tipo de carbohidrato en el concentrado sobre el consumo de alimento de vacas lecheras en pastoreo. Arch Med Vet 38, 123-128.

Pulido RG, S Berndt, P Orellana, F Wittwer. 2007. Effect of the source of carbohydrate in concentrate on the performance of high producing dairy cows during spring grazing. Arch Med Vet 39, 19-26.

Reis RB, DK Combs. 2000. Effects of increasing levels of grain supplementation on rumen environment and lactation performance of dairy cows grazing grass-legume pasture. J Dairy Sci 83, 2888-2898.

Rook AJ, CA Huckle, PD Penning. 1994. Effects of sward height and concentrate supplementation on the ingestive behaviour of springcalving dairy cows grazing grass-clover swards. Appl Anim Behav Sci 40, 101-112.

Rook AJ. 2000. Principles of foraging and grazing behaviour. In: Hopkins A (ed). Grass: Its Production and Utilization. Blackwell Science, Oxford, UK, Pp 229.

Sairanen A, H Khalili, P Virkajärvi. 2006. Concentrate supplementation responses of the pasture-fed dairy cow. Livestock Science 104, 292-302.

Sayers HJ, CS Mayne, CG Bartram. 2003. The effect of level and type of supplement offered to grazing dairy cows on herbage intake, 
animal performance and rumen fermentation characteristics. Anim Sci 76, 439-454.

Stockdale, CR. 2000. Levels of pasture substitution when concentrates are fed to grazing dairy cows in northern Victoria. Aust J Exp Agric 40, 913-921.
Tilley JMA, RA Terry. 1963. A two stage technique for in vitro digestion of forage crops. J Br Grassl Soc 18, 104-111.

Van Soest PJ, J Robertson, B Lewis. 1991. Methods for dietary fiber, neutral detergent fiber, and nonstarch polysaccharides in relation to animal nutrition. J Dairy Sci 74, 3583-3595. 
